\title{
Molecular Modeling of Acidic Treated PSTM-3T Polymer for Removal of Heavy Metal Ions by Experimental and Computational Studies
}

\author{
Natsagdorj Narantsogt, ${ }^{1}$ Gunchin Burmaa, ${ }^{2}$ Adiya Perlee-Oidov, ${ }^{1}$ \\ Nyamdorj Shurkhuu, ${ }^{3}$ and Namsrai Javkhlantugs ${ }^{3}$ \\ ${ }^{1}$ School of Natural Sciences, Mongolian State University of Education, 210648 Ulaanbaatar, Mongolia \\ ${ }^{2}$ Institute of Chemistry and Chemical Technology, Mongolian Academy of Science, 13330 Ulaanbaatar, Mongolia \\ ${ }^{3}$ Center for Nanoscience and Nanotechnology, School of Engineering and Applied Sciences, National University of Mongolia, \\ P.O. Box 46/290, Room 127, Main building, University Street 1, 14201 Ulaanbaatar, Mongolia
}

Correspondence should be addressed to Namsrai Javkhlantugs; javkhlantugs@num.edu.mn

Received 18 December 2013; Revised 7 March 2014; Accepted 9 March 2014; Published 26 May 2014

Academic Editor: Peng He

Copyright (C) 2014 Natsagdorj Narantsogt et al. This is an open access article distributed under the Creative Commons Attribution License, which permits unrestricted use, distribution, and reproduction in any medium, provided the original work is properly cited.

The synthesized poly[N, $\mathrm{N}^{\prime}$-bis(3-silsesquioxanilpropyl)-thiocarbamide] (PSTM-3T) was used and the surface morphology and microstructure of it were analyzed by scanning electron microscopy with energy dispersive spectrometer (SEM/EDS). The molecular structure change of the PSTM-3T polymer of the PSTM-3T after treatment by acidic solution with different pHs was revealed using FT-IR experiments and ab initio calculations with density functional theory method. The sorption efficiency of the heavy metal ions depends on the molecular structure change of PSTM-3T after treatment of different $\mathrm{pH}$ aqueous solutions. After the treatment of acidic solution $(\mathrm{pH}=2)$ of PSTM-3T, the polymer formed the tautomer state to increase the sorption efficiency for chromate ion. For the increment of $\mathrm{pH}$ value for acidic solution, the PSTM-3T polymer was dissociated to increase the sorption efficiency for copper ion.

\section{Introduction}

Industrial wastewater pollution from heavy metals is a major concern in developing countries. The industries produce the wastewater containing heavy metal ions with high concentrations. There exist many treatment techniques which have been developed such as chemical precipitation [1], adsorption $[2,3]$, and ion exchange [4].

Copper has been one of the most widely used metals for centuries and is mainly employed in electronic and electrical, electroplating, and mining industries. The organic-inorganic polymeric sorbents are known for their excellent surface characteristics for the effective adsorption of metals. For example, the preconcentration and separation of elements by using the chelating silicon-organic polymers have been reported [5-7]. However, most of chelating silicon polymers are used for preconcentration and determination of noble metal ions, and their synthesis of silica organic polymers usually takes a long time and synthetic process is complicated.

One of the other surface active simple polymers is poly[ $\mathrm{N}, \mathrm{N}^{\prime}$-bis(3-silsesquioxanilpropyl)-thiocarbamide] (PSTM-3T) which was synthesized [8] and investigated for determination of noble metals of gold, platinum, and palladium [9]. Previously, we revealed the adsorption kinetics for removal of $\mathrm{Cu}(\mathrm{II})$ and $\mathrm{Cr}(\mathrm{VI})$ ions from the wastewater using PSTM-3T polymer [10, 11]. In this study, we revealed the molecular structure change of PSTM-3T for sorption mechanism of heavy metal ions after treatment by different acidic solutions using frontier-transform infrared spectrophotometer (FT-IR) and ab initio calculations in detail. 


\section{Materials and Methods}

2.1. Synthesis of PSTM-3T. poly[N,N'-bis(3-silsesquioxanilpropyl)-thiocarbamide] (PSTM-3T) polymer (Scheme 1) was synthesized from silicon-organic monomer of N,N ${ }^{\prime}$-bis(3triethoxysilylpropyl)-thiocarbamide by hydrolytic polycondensation reaction in aqueous media with $\mathrm{pH}=8-9$ at the boiling temperature [8]. The synthesized PSTM-3T was characterized by scanning electron microscopy with energy dispersive spectrometer (SEM/EDS) and frontier-transform infrared (FT-IR) spectroscopy.

\subsection{Scanning Electron Microscopy (SEM) and Energy Disper-} sive Spectrometer (EDS). The surface morphology and microstructure were examined by scanning electron microscopy (Philips XL30, The Netherlands). The powdered samples were mounted onto double-sided carbon tapes and sputtercoated with palladium using a Cressington sputter coater 108A (Cressington Scientific Instruments, Watford, UK). The SEM images were taken on a Philips XL30 field emission gun SEM (FEI Company, Hillsboro, OR, USA) and EDS results were obtained using the built-in EDAX Genesis software.

2.3. Frontier-Transform Infrared (FT-IR) Spectroscopy. The chemical bond frequencies of functional groups of polymer before and after treatment by acidic solutions were analyzed by using frontier-transform infrared (FT-IR) spectrophotometer (IRPrestige-21, Shimadzu, Tokyo, Japan). The powdered samples were mixed with $\mathrm{KBr}$ to make the pellets. The FT-IR spectra were obtained with frequency range of 4000$400 \mathrm{~cm}^{-1}$.

2.4. Ultraviolet Visible (UV-Vis) Spectrophotometer. The amounts of metal ions in an initial solution and after treatment by polymer were analyzed by using ultraviolet visible (UV-vis) spectrophotometer (U-1000, HITACHI, Tokyo, Japan). The samples were prepared by mixing with the complexing reagent as diethyl dithiocarbamate for copper ion [12] and 1,5-diphenylcarbazide for chromate ion [13] which forms a color with metal ion. After being filtered, all samples were analyzed using quartz cuvette with a path length of $10 \mathrm{~mm}$ at wavelength of $430 \mathrm{~nm}$ for copper ion and $540 \mathrm{~nm}$ for chromate ion. The spectrophotometer measurement for sorption kinetics of the PSTM-3T at different $\mathrm{pHs}$ was carried out with stirrer/hot plate (Corning PC-620D) after treatment of $0.05 \mathrm{~g}$ PSTM-3T into $50 \mathrm{~mL}$ (20 mg/L of copper and $60 \mathrm{mg} / \mathrm{L}$ chromate ions) solution.

2.5. Computational Calculation Procedure. The molecular structures of PSTM-3T polymer were modeled at different $\mathrm{pH}$ conditions with consideration of tautomerization and dissociation. Geometries of all the considered model molecules (Figure 1) in this study were fully optimized by using density functional theory (DFT) with Becke's three-parameter hybrid exchange function [14] and the Lee-Yang-Parr correlation function (B3LYP) [15] and with the 6-31G $(\mathrm{d}, \mathrm{p})$ basis set [16] method in Gaussian 03 package [17]. Vibrational frequencies and absolute IR intensities of optimized structures were

$$
\left(\mathrm{O}_{1.5} \mathrm{Si}-\left(\mathrm{CH}_{2}\right)_{3}-\mathrm{NH}-\underset{\mathrm{S}}{\mathrm{C}}-\mathrm{NH}-\left(\mathrm{CH}_{2}\right)_{3}-\mathrm{SiO}_{1.5}\right)_{n}
$$

Scheme 1: Representation of molecular structure of PSTM-3T polymer.

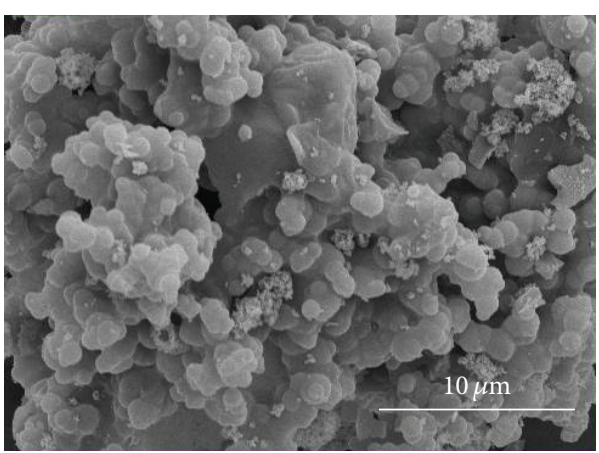

FIGURE 1: SEM image of synthesized PSTM-3T polymer.

investigated with 6-311G(d, p) level. All the convergent precisions were the system default values, and the all calculations were carried out on the standard lab-level workstation with AMD Opteron 285 dual core CPU. Data visualization was carried out using Gauss View 3.0 [18].

\section{Results and Discussion}

3.1. Characterization of PSTM-3T Polymer. The details of chemical composition of PSTM-3T by EDS analysis are given in Table 1. The major elements of the sample verified the PSTM-3T polymer. The elemental analysis showed the similar results with theoretically calculated contents of synthesized title polymer as formula of $\mathrm{C}_{7} \mathrm{H}_{14} \mathrm{~N}_{2} \mathrm{O}_{3} \mathrm{SSi}_{2}$ (Scheme 1). The SEM was performed on an irregular and porous surface (Figure 1) which indicates high surface areas of $490 \mathrm{~m}^{2} / \mathrm{g}$ with $3.53 \mathrm{~cm}^{3} / \mathrm{g}$ total pore volume.

The functional groups were identified using FT-IR experiment and the spectrum is shown in Figure 2. In Figure 2, the observable IR signals for PSTM-3T were 580.57 and $692.41 \mathrm{~cm}^{-1}$ for C-S, $1560.41,3373.50$, and $3388.93 \mathrm{~cm}^{-1}$ for $\mathrm{N}-$ $\mathrm{H}$, and $1631.78 \mathrm{~cm}^{-1}$ for C-N. The IR signals were similar with the group frequencies [19]. In this study, the model of PSTM3T (Figure 3(a)) was investigated using the density functional B3LYP method with 6-311G(d, p) basis set (Table 2). All the values have relative deviation less than $5.3 \%$ (Table 2) and the theoretical values are in good agreement with experimental values, the same as previous other reports [20].

3.2. Sorption of Heavy Metal Ions. The solution $\mathrm{pH}$ is one of the most important factors for heavy metal sorption from the aqueous solution. Sorption of copper and chromate ions was revealed at various $\mathrm{pH}$ conditions and the sorption strengths are shown in Figure 4. The fraction of sorption of copper ion as determined by the removal of copper ion percentage 
TABLE 1: Chemical composition of PSTM-3T and after treatment into ion solutions.

\begin{tabular}{lccc}
\hline Elements & Weight [\%] & \\
\hline $\mathrm{C}$ & 36.94 & 34.33 & $\mathrm{Cr}(\mathrm{VI})$ \\
$\mathrm{N}$ & 14.23 & 9.71 & 37.42 \\
$\mathrm{O}$ & 23.16 & 22.67 & 7.73 \\
$\mathrm{Si}$ & 18.40 & 13.00 & 21.66 \\
$\mathrm{~S}$ & 7.10 & 10.51 & 21.75 \\
$\mathrm{Cu}$ & & 0.52 & 11.17 \\
$\mathrm{Cr}$ & & & \\
\hline
\end{tabular}

TABLE 2: The comparison of experimental (in Figure 2) and theoretical frequencies of PSTM-3T. The percentage of relative deviation of model in different frequencies is shown in parenthesis.

\begin{tabular}{lccc}
\hline \multirow{2}{*}{ Groups } & \multicolumn{2}{c}{ Wavenumbers $[1 / \mathrm{cm}]$} & \multirow{2}{*}[\%]{} \\
& Exp. & Cal. & -3.09 \\
CS & 580.57 & 562.64 & +5.22 \\
\hline \multirow{2}{*}{$\mathrm{NH}$} & 692.41 & 728.57 & -1.13 \\
& 1560.41 & 1542.81 & +5.29 \\
& 3373.50 & 3552.03 & +5.06 \\
\hline $\mathrm{CN}$ & 3388.93 & 3560.54 & -4.01 \\
\hline
\end{tabular}

relative to initial concentration by PSTM-3T at various $\mathrm{pHs}$ was calculated as (1)

$$
F=\frac{\left(A_{0}-A_{t}\right)}{A_{0}}
$$

where $A_{0}$ and $A_{t}$ are the initial and time absorbance of the copper ion. From Figure 4(a), the removal efficiency of copper ion at $\mathrm{pH}=5$ is clearly seen higher than at other lower $\mathrm{pH}$ conditions. The equilibrium amounts of copper ion onto PSTM-3T surface at different $\mathrm{pH}$ conditions were calculated as (2)

$$
Q_{e}=\frac{\left(C_{0}-C_{e}\right) V}{m}
$$

where $Q_{e}(\mathrm{mg} / \mathrm{g})$ is adsorbed equilibrium amount of the ion per unit mass of PSTM-3T polymer. $C_{0}$ and $C_{e}$ are initial and equilibrium ion concentration $(\mathrm{mg} / \mathrm{L})$, respectively. $V$ is the volume of solution and $m$ is the mass (g) of the PSTM-3T polymer.

The equilibrium amounts of chromate ion onto PSTM$3 \mathrm{~T}$ surface were investigated at different $\mathrm{pH}$ conditions which are shown in Figure 4(b). From Figure 4(b), the removal of chromate ion at $\mathrm{pH}=2$ can be seen higher than at other $\mathrm{pH}$ conditions.

3.3. Molecular Modeling at Different pHs. The EDS analysis after treatment of PSTM-3T into copper and chromate ion solutions at $\mathrm{pH} 5$ and $\mathrm{pH} 2$, respectively, was analyzed and these $\mathrm{pH}$ conditions were higher removal efficiencies for each ion which were discussed in previous section (Figure 4).

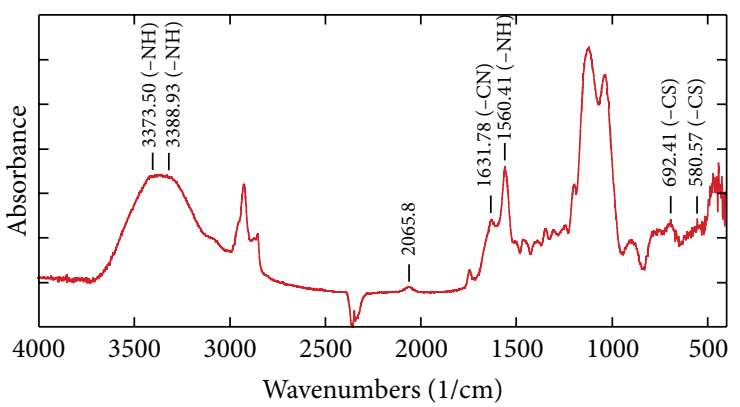

FIGURE 2: FT-IR spectra of PSTM-3T polymer.

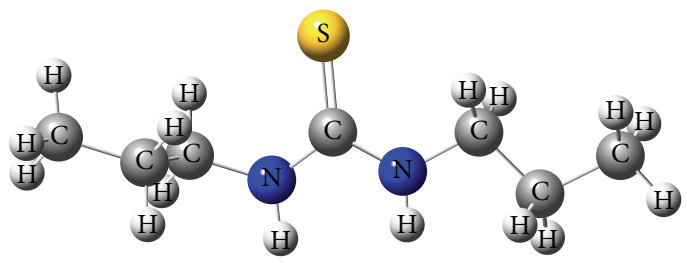

(a)

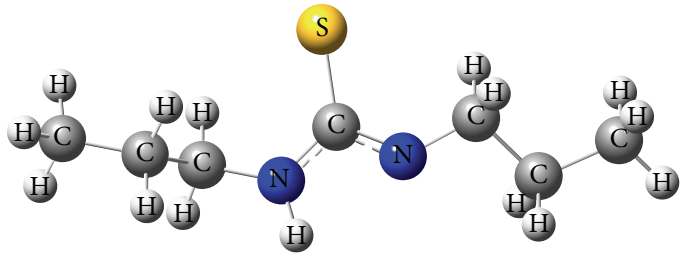

(b)

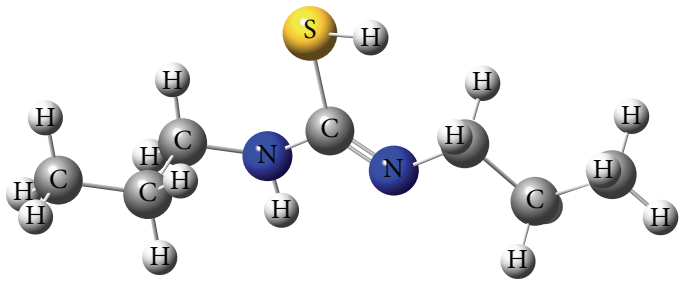

(c)

FIGURE 3: Optimized molecular model structures of PSTM-3T polymer before (a) and after treatment by $\mathrm{pH}=5$ (b) and $\mathrm{pH}=2$ (c) solutions. Carbon, hydrogen, nitrogen, and sulfur atoms are shown as gray, silver, blue, and yellow ball representations, respectively.

The copper and chromium atoms were obtained in PSTM3T polymer using EDS elemental analysis after treatment of PSTM-3T into ion solutions and the weight percentage of them was shown in Table 1.

The FT-IR spectra of PSTM-3T after treatment by acidic solutions are shown in Figure 5. In Figure 5(a), the IR signals for PSTM-3T at pH5 were found at 553.57 and $665.44 \mathrm{~cm}^{-1}$ for C-S, 1558.48 and $3300.20 \mathrm{~cm}^{-1}$ for N-H, and $1633.71 \mathrm{~cm}^{-1}$ for C-N.

The functional group from protonated and unprotonated states depends on the solution $\mathrm{pH}$ values. Therefore, we considered the additional two models of the dissociated (Figure 3(b), Scheme 2) and tautomer (Figure 3(c), 


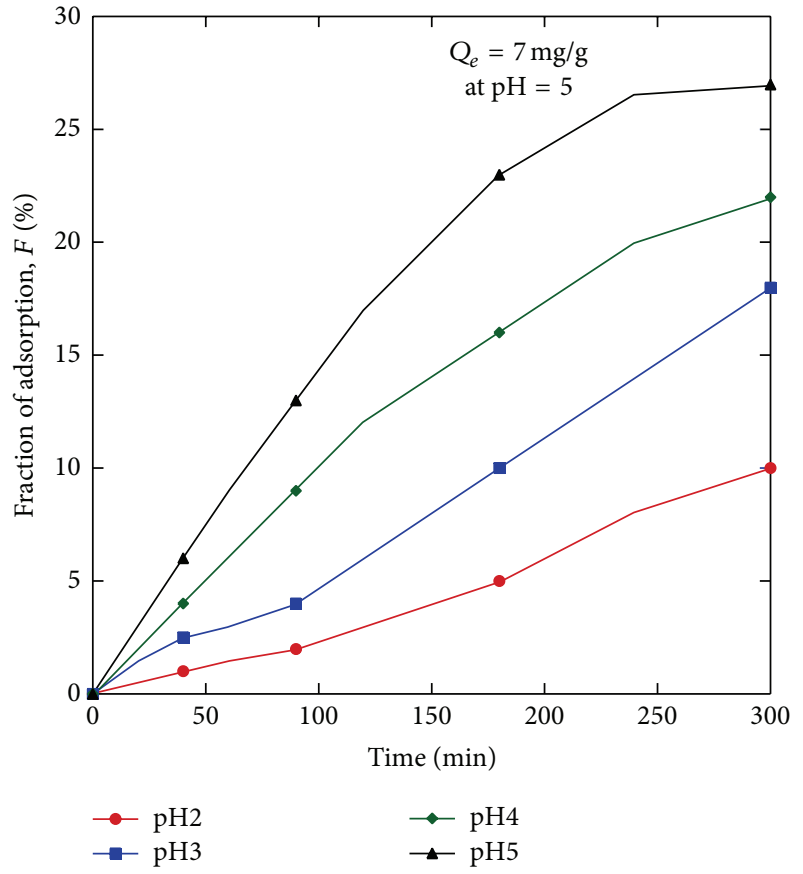

(a)

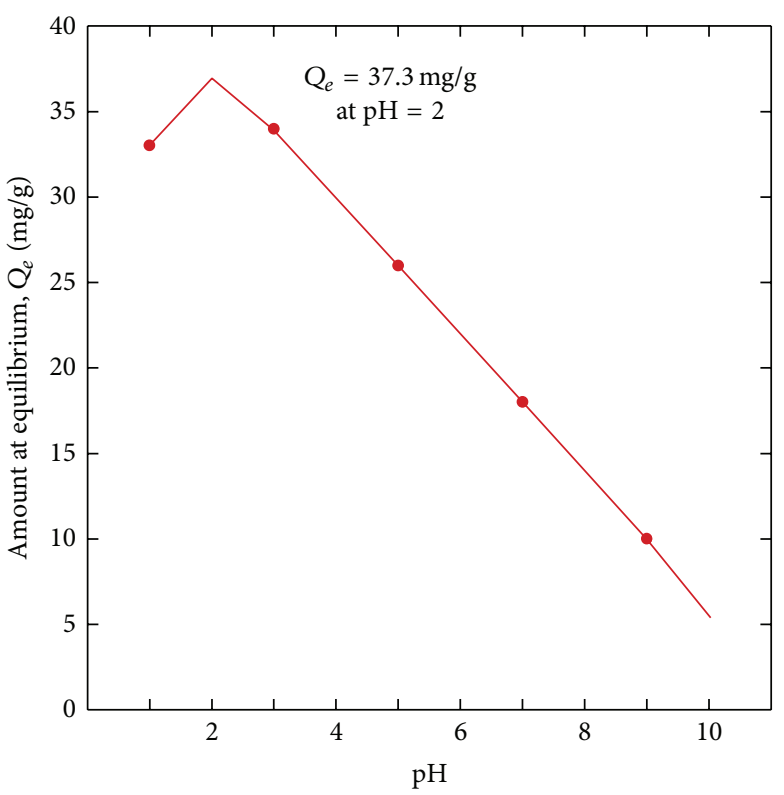

(b)

FIGURE 4: Sorption efficiency of copper ion (a) and equilibrium amount of chromate ion per unit mass of polymer (b) at different pHs.

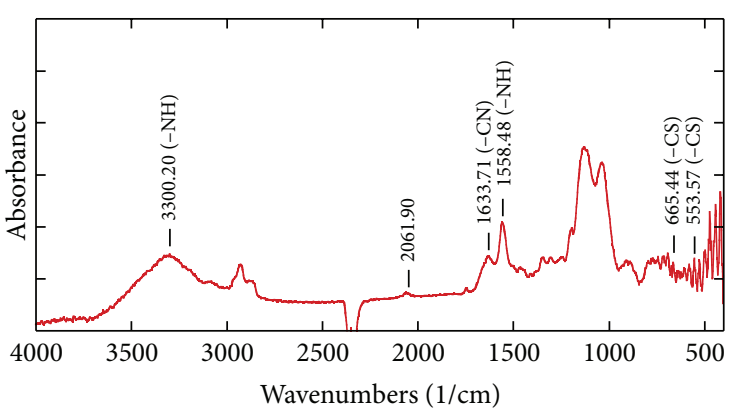

(a)

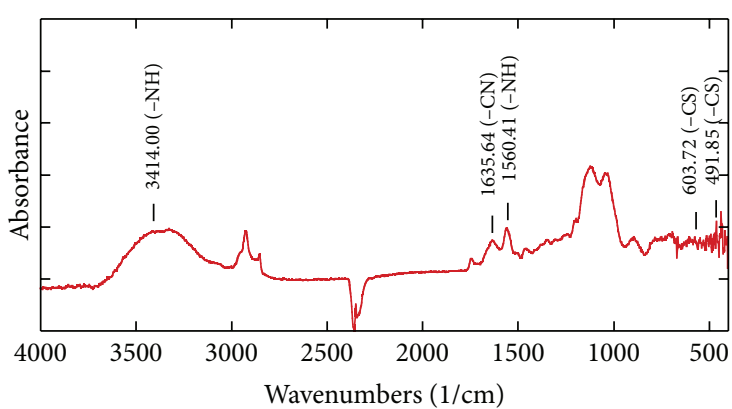

(b)

FIGURE 5: FT-IR spectrum of PSTM-3T polymer after treatment by $\mathrm{pH}=5$ (a) and $\mathrm{pH}=2$ (b) acidic solutions.

Scheme 2) states and calculated the frequencies of these two models which are shown in Tables 3 and 4 . The model of dissociated state of PSTM-3T (Figure 3(b), Scheme 2) was investigated using the density functional B3LYP method with 6-311G(d, p) level. The major values have relative deviation less than 6.5\% (Table 3) and the theoretical values are in good agreement with experimental values. In Figure 5(a), the frequency of $2061.90 \mathrm{~cm}^{-1}$ was obtained experimentally. Also in spectra of PSTM-3T polymer (Figure 2), the frequency of $2065.8 \mathrm{~cm}^{-1}$ for $\mathrm{C}=\mathrm{S}$ state was obtained, the same as at $\mathrm{pH}=5$. Previously, Grebenev et al. investigated $\mathrm{OC}=\mathrm{S}$ with $\mathrm{H}_{2}, \mathrm{HD}$, and $\mathrm{D}_{2}$ complexes using high-resolution IR spectroscopy and found that the main differences between the spectra of them are near the frequency around $2061.0 \mathrm{~cm}^{-1}$ [21]. We calculated single molecule in vacuum; therefore, the frequency of interbond frequency was not obtained theoretically.

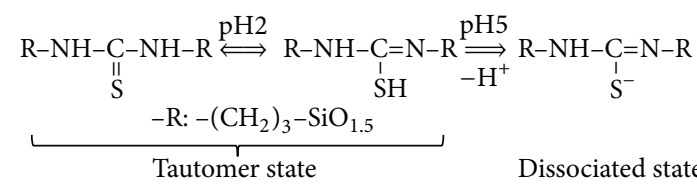

SCHEME 2: Representation of molecular model structures of tautomer and dissociated states.

For sorption of chromate ion at $\mathrm{pH}=2$, Figure 5(b) showed that the IR signals for PSTM-3T were found at 491.85 and $603.72 \mathrm{~cm}^{-1}$ for C-S, 1560.41 and $3414.00 \mathrm{~cm}^{-1}$ for $\mathrm{N}-\mathrm{H}$, and $1635.29 \mathrm{~cm}^{-1}$ for C-N. The model of tautomer state of PSTM-3T (Figure 3(c), Scheme 2) was investigated using the density functional B3LYP method with 6-311G(d, p) level. All the values have relative deviation less than 5.2\% (Table 4) and the theoretical values are in good agreement 
TABLE 3: The comparison of experimental (in Figure 5) and theoretical (in Figure 3(b)) frequencies of PSTM-3T after treatment by nitric acid $(\mathrm{pH}=5)$ solution. The percentage of relative deviation of model in different frequencies is shown in parenthesis.

\begin{tabular}{lccc}
\hline \multirow{2}{*}{ Groups } & \multicolumn{2}{c}{ Wavenumbers $[1 / \mathrm{cm}]$} & \multirow{2}{*}[\%]{} \\
& Exp. & Cal. & +5.20 \\
\multirow{2}{*}{$\mathrm{CS}$} & 553.57 & 582.38 & +4.14 \\
\hline \multirow{2}{*}{$\mathrm{NH}$} & 665.44 & 692.99 & -1.95 \\
& 1558.48 & 1528.08 & +8.36 \\
\hline Inter & 3300.20 & 3575.94 & \\
\hline $\mathrm{CN}$ & 2061.90 & & -6.47 \\
\hline
\end{tabular}

TABle 4: The comparison of experimental (in Figure 5) and theoretical (in Figure 3(c)) frequencies of PSTM-3T after treatment by hydrochloric acid $(\mathrm{pH}=2)$ solution. The percentage of relative deviation of model in different frequencies is shown in parenthesis.

\begin{tabular}{lccc}
\hline \multirow{2}{*}{ Groups } & \multicolumn{2}{c}{ Wavenumbers $[1 / \mathrm{cm}]$} & \multirow{2}{*}{ Exp] } \\
& Exp. & Cal. & +5.21 \\
$\mathrm{C}$ & 491.85 & 517.48 & -0.09 \\
\hline \multirow{2}{*}{$\mathrm{NH}$} & 603.72 & 603.19 & -4.94 \\
& 1560.41 & 1483.39 & +3.98 \\
\hline $\mathrm{SH}$ & 3414.00 & 3549.76 & \\
\hline $\mathrm{CN}$ & & 2700.03 & +3.12 \\
\hline
\end{tabular}

with experimental values. Theoretically, the frequency of functional group of - $\mathrm{SH}$ was obtained as $2700.03 \mathrm{~cm}^{-1}$ which was not determined by FT-IR experiment because it may have very weak absorbance in nature.

Finally, the PSTM-3T polymer forms dissociated state with thiolate ion when the highest sorption for copper ion occurs at $\mathrm{pH}=5$. The copper ion strongly interacts with opposite charge of thiolate ions for sorption efficiency. The tautomer state of polymer with $-\mathrm{SH}$ group at $\mathrm{pH}=2$ may activate the reduction reaction for chromate ion.

\section{Conclusion}

The molecular structure change of the PSTM-3T polymer for removal of heavy metal ions of the PSTM-3T after treatment by acidic solution with different $\mathrm{pHs}$ was clearly determined using FT-IR experiments and $a b$ initio calculations. After the treatment of acidic solution $(\mathrm{pH}=2)$ of PSTM-3T, the polymer formed the tautomer state to increase the sorption efficiency for chromate ion. For the increment of $\mathrm{pH}$ value for acidic solution, the PSTM-3T polymer was dissociated to increase the sorption efficiency for copper ion. Finally, decrement of $\mathrm{pH}$ value may increase the sorption efficiency for metal anions by reduction reaction.

\section{Conflict of Interests}

The authors declare that there is no conflict of interests regarding the publication of this paper.

\section{Acknowledgments}

The authors are grateful to the National University of Mongolia and Yokohama National University for their help under the Research Agreement between them for the calculations. Namsrai Javkhlantugs thanks, for the part of calculations, The Asian Research Center in Mongolia and the Korean Foundation for Advanced Studies within the framework of the Project no. 1 (2014-2015).

\section{References}

[1] M. M. Matlock, B. S. Howerton, and D. A. Atwood, "Irreversible precipitation of mercury and lead," Journal of Hazardous Materials, vol. 84, no. 1, pp. 73-82, 2001.

[2] D. Mohan and S. Chander, "Removal and recovery of metal ions from acid mine drainage using lignite-a low cost sorbent," Journal of Hazardous Materials, vol. 137, no. 3, pp. 1545-1553, 2006.

[3] V. K. Gupta and S. Sharma, "Removal of cadmium and zinc from aqueous solutions using red mud," Environmental Science and Technology, vol. 36, no. 16, pp. 3612-3617, 2002.

[4] V. J. Inglezakis, M. A. Stylianou, D. Gkantzou, and M. D. Loizidou, "Removal of $\mathrm{Pb}$ (II) from aqueous solutions by using clinoptilolite and bentonite as adsorbents," Desalination, vol. 210, no. 1-3, pp. 248-256, 2007.

[5] R. K. Sharma, "Design, synthesis, and application of chelating polymers for separation and determination of trace and toxic metal ions. A green analytical method," Pure and Applied Chemistry, vol. 73, no. 1, pp. 181-186, 2001.

[6] M. Zougagh, J. M. Cano Pavón, and A. Garcia De Torres, "Chelating sorbents based on silica gel and their application in atomic spectrometry," Analytical and Bioanalytical Chemistry, vol. 381, no. 6, pp. 1103-1113, 2005.

[7] M. E. Sears, "Chelation: Harnessing and enhancing heavy metal detoxification-a review," The Scientific World Journal, vol. 2013, Article ID 219840, 13 pages, 2013.

[8] M. G. Voronkov, N. N. Vlasova, Y. H. Pozhidaev, A. E. Pestunovich, and A. I. Kirillov, "High effective complicit and ampelite-poly[N,N'-bis(3-silsesquiocsanilpropyl)-thiocarbamide]," Doklady Akademii Nauk SSSR, vol. 320, no. 3, pp. 658-662, 1991 (Russian).

[9] Y. Pozhidaev, N. Vlasova, I. Vasilyeva, and M. Voronkov, "Determination of noble metals in rocks and ores using adsorbent PSTM-3T," Advanced Science Letters, vol. 19, no. 2, pp. 615-618, 2013.

[10] N. Narantsogt, G. Burmaa, O. Nasantogtokh, and A. PerleeOidov, "Adsorption kinetics for the removal of copper(II) from aqueous solution by adsorbent PSTM-3T," Mongolian Journal of Chemistry, vol. 12, pp. 1-6, 2011.

[11] G. Burmaa, N. Narantsogt, O. Nasantogtokh, and Perlee-Oidov, "Chromium(VI) sorption from aqueous solution by siliconorganic sorbent contaiting thicarbamide groups," Mongolian Journal of Chemistry and Chemical Engineering, vol. 1, pp. 7-12, 2012.

[12] G. A. Shar and M. I. Bhanger, "Spectrophotometric determination of copper (II) with diethyldithiocarbamate in polyoxyethylenedodecylether (Brij-35) media," Journal of the Chemical Society of Pakistan, vol. 24, no. 3, pp. 185-189, 2002. 
[13] M. Noroozifar and M. Khorasani-Motlagh, "Specific extraction of chromium as tetrabutylammonium-chromate and spectrophotometric determination by diphenylcarbazide: speciation of chromium in effluent streams," Analytical Sciences, vol. 19, no. 5, pp. 705-708, 2003.

[14] A. D. Becke, "Density-functional thermochemistry. III. The role of exact exchange," The Journal of Chemical Physics, vol. 98, no. 7, pp. 5648-5652, 1993.

[15] C. Lee, W. Yang, and R. G. Parr, "Development of the ColleSalvetti correlation-energy formula into a functional of the electron density," Physical Review B, vol. 37, no. 2, pp. 785-789, 1988.

[16] W. J. Hehre, L. Radom, P. R. Schleyer, and J. A. Pople, Ab Initio Molecular Orbital Theory, John Wiley \& Sons, New York, NY, USA, 1987.

[17] M. J. Frisch, G. W. Trucks, H. B. Schlegel et al., Gaussian 03, Revision C.01, Gaussian Inc., Wallingford, Conn, USA, 2004.

[18] A. Frish, R. D. Dennington II, T. A. Keith et al., GaussView Version 4.1, Gaussian Inc., Wallingford, Conn, USA, 2007.

[19] J. Coates, "Interpretation of infrared spectra, a practical approach," in Encyclopedia of Analytical Chemistry, R. A. Meyers, Ed., pp. 10815-10837, Jonh Wiley \& Sons, Chichester, UK, 2004.

[20] M. Pousti, M. Abbaszadeh, and M. S. Iashkenari, "A combined experimental and theoretical studies on molecular structure and vibrational spectra of polyaniline and polyaniline/silver nanocomposite," Synthetic Metals, vol. 183, pp. 63-68, 2013.

[21] S. Grebenev, B. G. Sartakov, J. P. Toennies, and A. F. Vilesov, "High-resolution infrared spectra of the OCS-H2, -HD, and D2 van der Waals complexes in liquid helium droplets," Journal of Chemical Physics, vol. 118, no. 19, pp. 8656-8670, 2003. 

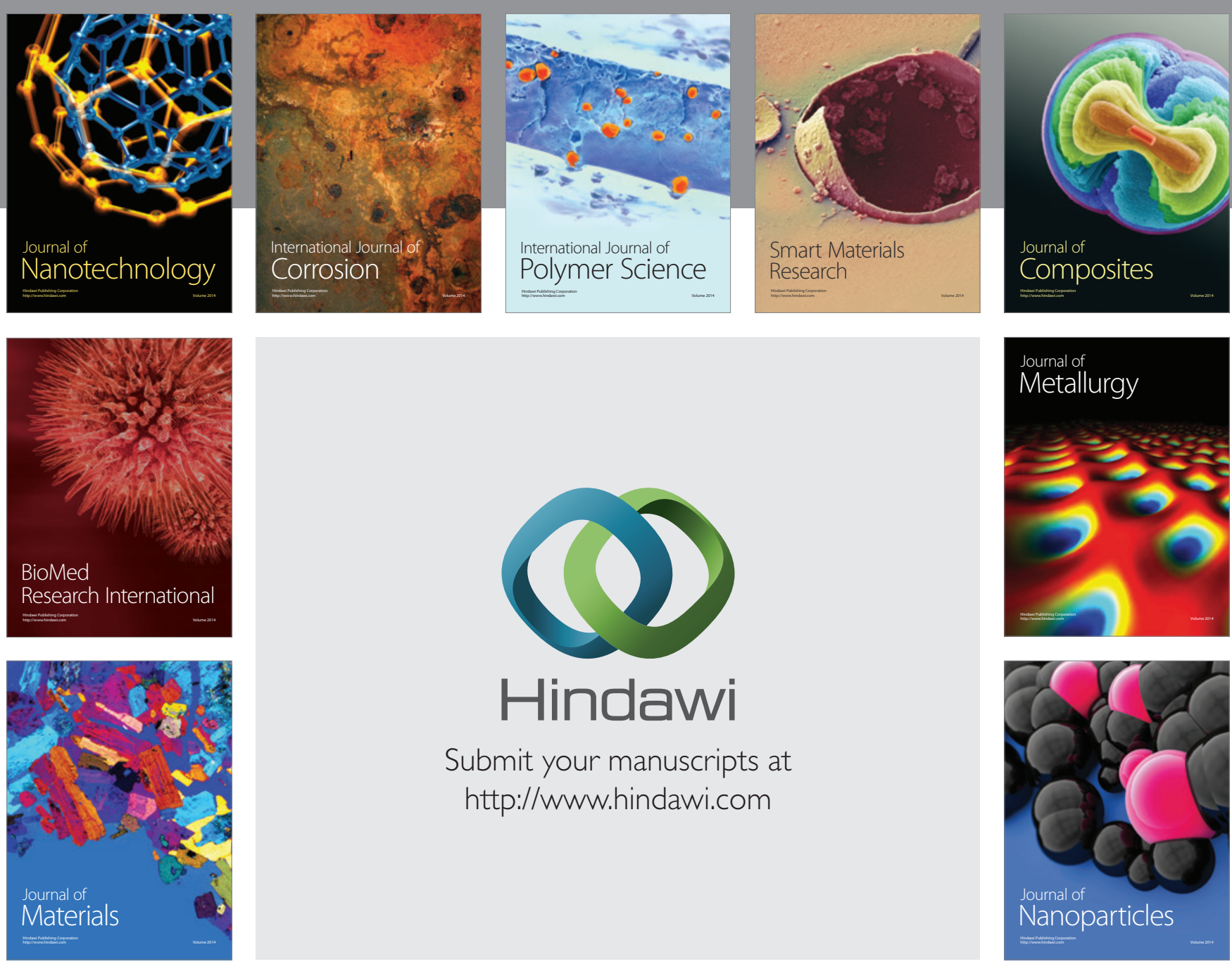

Submit your manuscripts at http://www.hindawi.com
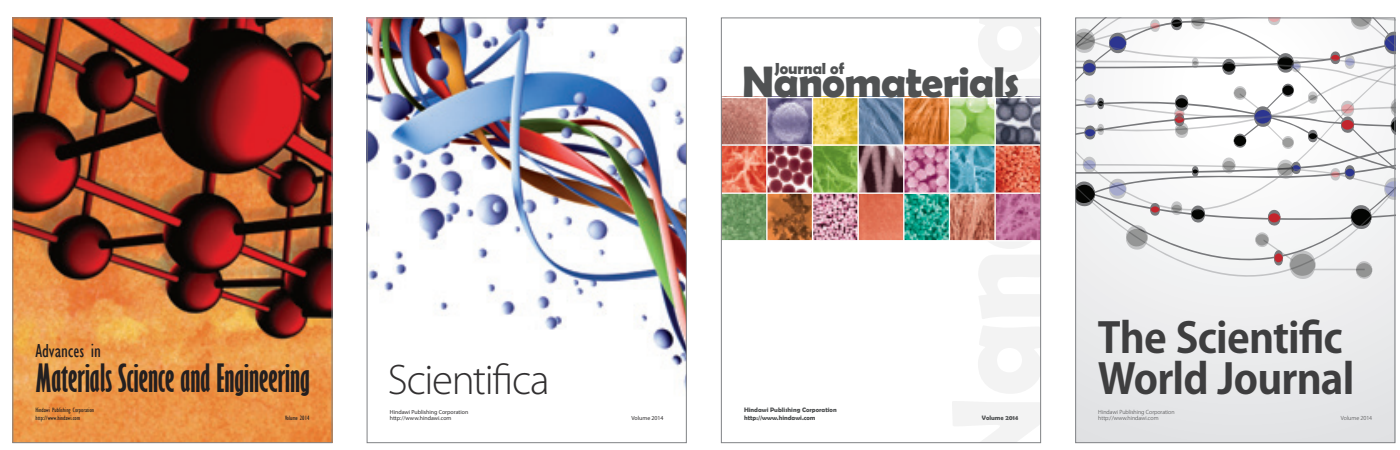

\section{The Scientific World Journal}
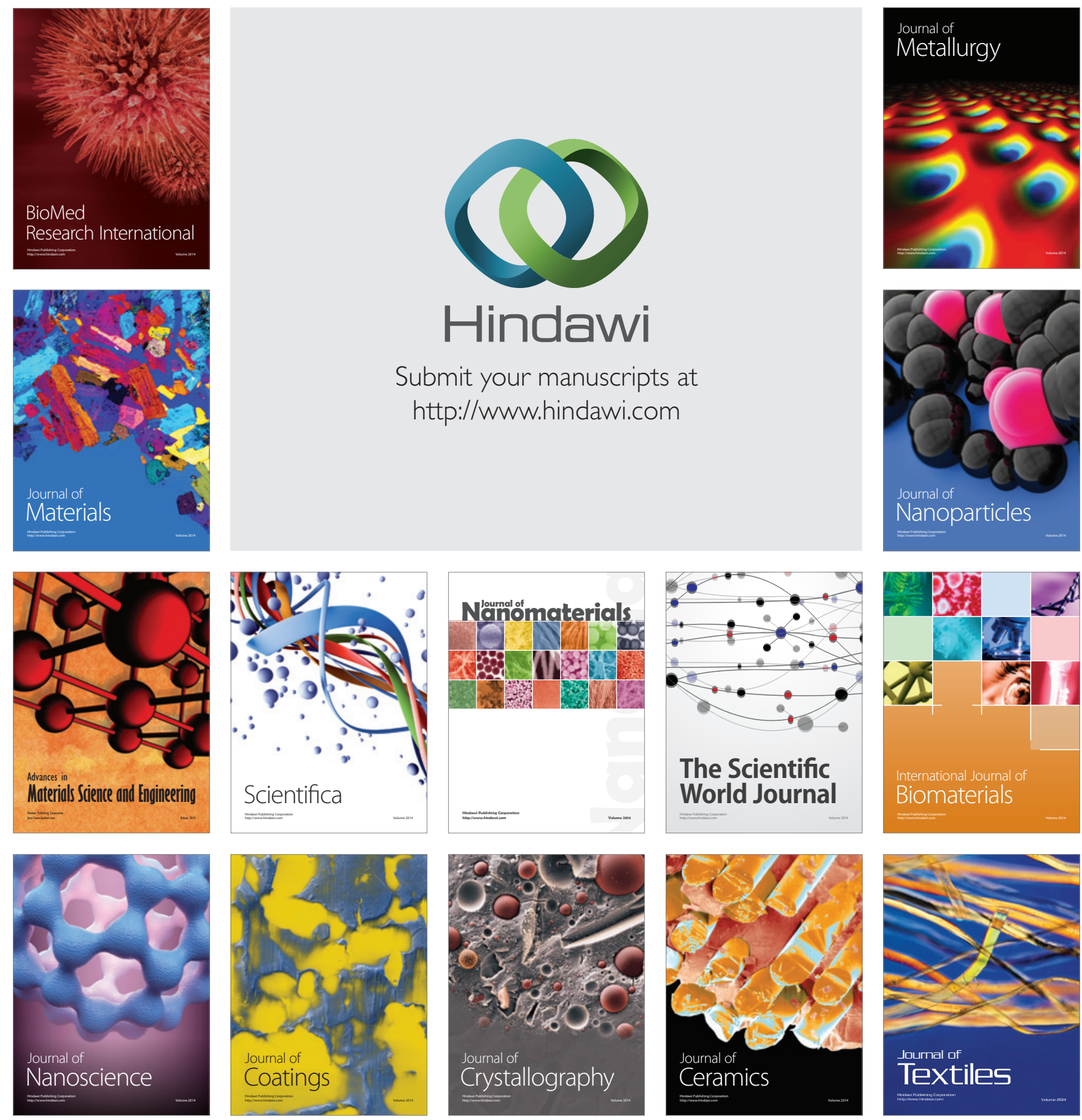